\title{
Idiopathic slow transit constipation is rare
}

\section{But delayed passage of meconium is common in the constipation clinic}

\author{
G. D. H. Croaker $\cdot$ R. Pearce $\cdot$ J. Li $\cdot$ I. Nahon $\cdot$ A. Javaid $\cdot$ Z. Kecskes
}

Published online: 31 October 2007

(C) Springer-Verlag 2007

\begin{abstract}
We hypothesise that constipated children would be more likely to come from a socially deprived background. We also hypothesise that a percentage of children with resistant constipation would have a congenital gut motility problem that might be recognised at birth, and that some of these would have slow transit constipation that could be recognised on nuclear transit study. One hundred and forty children with a constipation related diagnosis were seen in the last 4 years, and were reviewed as a retrospective audit. Twenty-six children who were felt likely to have a congenital cause for their constipation were offered nuclear colon transit study to search for slow transit constipation. One hundred and forty children from the constipation clinic were reviewed. There were 67 females (47.9\%) and 73 males (52.1\%), a sex ratio near equality. The mean age at presentation was 5.38 years. Forty-one percent were formally discharged, $36 \%$ were lost to follow up, and $23 \%$ are still being seen. There was a highly significant tendency for these children to have delayed passage of meconium, as compared normal newborns $(P<<0.001)$. Twenty-six children were considered for possible transit study, and 14 were performed. Four of these were normal, seven showed hold up in the recto-sigmoid, and three showed more proximal
\end{abstract}

\footnotetext{
G. D. H. Croaker $(\bowtie) \cdot$ R. Pearce · I. Nahon · A. Javaid .

Z. Kecskes

The Canberra Hospital, Garran,

ACT 2605, Australia

e-mail: croakers@netspeed.com.au

G. D. H. Croaker · J. Li · Z. Kecskes

The Australian National University,

Canberra, Australia
}

slow transit. Two of these probably have non ISTC diagnoses. Social class seems similar to the general population on the criterion employed. Delayed passage of meconium in this group was significantly more frequent than in the general population, but only one of the group seems likely to have truly idiopathic slow transit constipation, and he did not have delayed passage of meconium. There is no evidence for an effect of social class in this population. Idiopathic slow transit constipation itself is rare.

\section{Background}

Constipation is a common problem, with high rates of presentation around the world. Constipation itself is a symptom, not a diagnosis, and individualising treatment is expected to yield quicker and more effective results than current methods involving often prolonged and frequently ineffective laxatives.

We have previously noted that children with neonatal obstruction sufficient to warrant a rectal suction biopsy are frequently still symptomatic a year later. We were curious as to whether this relation between neonatal symptoms and constipation noted prospectively would be important when reviewed retrospectively, and whether there would be objective correlates of delayed motility in these children on nuclear transit study.

ISTC has recently been proposed as a potentially treatable cause of constipation in a percentage of constipated children $[1,2]$. With a view to seeing, and perhaps treating any of these children in our clinic we began offering nuclear transit studies to children with intractable constipation 18 months ago. The audit of the clinic and the 
results of the nuclear medicine colon transit studies form the substance of this report.

\section{Materials and methods}

Clinic letters coded for constipation by the first author over four years were reviewed. If the child was born in the Canberra Hospital, birth records were reviewed to reliably establish the onset of defaecation. For all other children, this data was obtained where possible as part of the standard clinical history. Other data included the age of presentation, the sex, the suburb of residence, the length of follow up, and other associated conditions. Cases with a pre-existing diagnosis such as Hirschsprung disease, anal stenosis, coeliac disease, cow's milk protein intolerance, amongst others, were noted. Delay in passage of meconium was compared with the same data obtained from the same institution on 700 normal neonates of varying gestations as part of another study, so far unpublished ("Normal values for first passage of meconium in neonates: evidence that the human GI tract continues to mature up to term." G. D. H. Croaker, U. Majeed et al. Presented at British Association of Paediatric Surgeons (BAPS) annual meeting 2004).

De-identified data was compared by suburb of residence at time of presentation between the constipation patients and a control group of children having a biopsy for coeliac disease. The mean real estate values as obtained from a real estate website were compared between groups, as a proxy for social status.

Children with a normal rectal biopsy (H and $\mathrm{E}$ stain), and failure to respond to supervised medical treatment for at least 6 months, or a history going back to the neonatal period were considered for study. Those with a strong family history of unremitting constipation and a prolonged period of failure of medical treatment were also considered. In most cases rectal biopsy, and/or ano-rectal manometry was also performed.

Nuclear colonic transit studies were performed off medication, and in some instances after the bowel had been emptied by gavage with polyethylene glycol solution or other means. The transit study itself was performed using standard gamma camera imaging procedures after ingestion of $8 \mathrm{MBq}$ gallium 67 citrate solution. Serial images were then taken using an appropriately collimated camera out to $96 \mathrm{~h}$ after ingestion. Areas of interest were marked, and activity remaining at each interval calculated. Registration of images was assisted by placement of a radioactive skin marker on the xiphisternum. The patient was advised to eat and otherwise live normally between scanning episodes. Passage of stools during the study was noted, and commented on in the final report.

\section{Results}

1. One hundred and forty constipated patients were identified. The average age at presentation was 5.38 years. There were 67 females $(47.9 \%)$ and 73 males $(52.1 \%)$, a sex ratio near equality.

Females presented slightly earlier than males (4.92 years, against 5.81 years) but this was not a significant difference. Overall at least one third of the families reported concern with soiling as well as constipation.

2. Fifty seven children were formally discharged from clinic apparently well during the four years, at an average of 9.53 months after the first clinic visit. Fifty one were lost to follow up after an average of 3.85 months from first visit, and 32 are still being seen. Age at last clinic visit was similar whether formally discharged, or lost to follow up.

3. Out of these 140 , there were 26 who were felt to be candidates for nuclear medicine colon transit study. Of the 12 who did not proceed to study, seven were getting better at the time of last contact, and one was actually discharged. One of these seven improving patients was noted to have an increase in number and size of nerve trunks on rectal suction biopsy, and to have a lower than normal recto-anal inhibitory response, and somewhat low internal anal sphincter pressure. We felt that he likely had a form of IND, but he has confounded us by improving without specific treatment.

Six have been lost to follow up, although two had been improving at last contact. One went to an ACE procedure, and declined further investigation, even though management of the ACE washout regime has been very difficult, with disappointing results.

To summarise those who did not proceed to transit study: nine were improving at last contact, one went to an ACE procedure and declined further investigation, and two who were lost to follow up did not seem to be improving at last contact.

At the time of writing, 14 have completed nuclear medicine transit studies. Those who proceeded to transit study were significantly more likely to be male (11 male, 3 female), compared with those who did not proceed (6 male, 6 female. $P=0.01$ by $\chi^{2}$ ). The age at presentation was the same for those who proceeded to study, as for those who did not -4.7 years.

There were three who had definitely abnormal colonic transit, two who were completely normal, and seven who had tracer retention in the recto-sigmoid region.

Of the three children with definitely abnormal transit study, two had no delay in passage of meconium when compared with gestation, and the neonatal history of one is unknown. All three are male. Two of the three with 
evident colonic motility abnormalities probably have non ISTC diagnoses. Probably only one of 140 referred patients has ISTC. A rate of 7.1 per 1,000 (95\% CI 0.2 to 39.2 per 1,000) using the exact method [3] (Even if we accept that the true rate includes all $3 / 140$, then the rate is still only $2.85 \%$. [95\% CI 4.4 to 61.3 per 1,000$]$ ).

4. For children born at or near term, average delay in passage of meconium was greater for those seen in the constipation clinic than for the normal population. There was a highly significant tendency for children in the constipation group to have reported a delay in passage of meconium of greater than $24 \mathrm{~h}$. Examination of the clinic notes shows that 12 of 88 where the history was available from the parents, reported delay in passage of meconium. In 43 cases the obstetric record was available to be interrogated from the hospital record. Records where a definite time interval was recorded (delivery room or post natal ward records where possible, or in two cases where the parents had definite information), showed that in fact 15 of 43 had delay greater than or equal to $24 \mathrm{~h}$. Combining the groupss (clinic notes and obstetric notes) we had 16 of 140 with delay in passage of meconium greater than $24 \mathrm{~h}$. Seventy-two were said to have had no delay, and 52 of 140 had an unknown interval till passage of meconium. Thirteen of the children with delayed passage of meconium were born at 37 weeks or more gestation. Of 88 where there is information on passage of meconium, 16 were delayed. Taking the smaller group of 43 where accurate data was available we see that 13 with delay $>24 \mathrm{~h}$ were born at term $(\geq 37$ weeks gestation). Comparing this with a group of normal children from this hospital's neonatal database, we see that amongst unselected children, only one of
259 children born at term had delay in passage of meconium $\geq 24 \mathrm{~h}$. Allowing for significant numbers of patients where data is missing, nonetheless the difference in incidence is highly significant considered both as a fraction of the whole (13/140), or of the group where obstetric records were available (13/43) $(P<<0.001$ in either case by chi square). There was no Hirschsprung disease in either group. Three of the 16 delayed meconium children were born pre-term. Two of these, born at 32 and 36 weeks had delay greater than usual for their degree of prematurity. The third was born at 26 weeks, and developed intestinal perforation soon after birth, but nonetheless had a degree of delay consistent with gestation, and probably should not be considered delayed in context.

5. Children with greater delay in passage of meconium had a tendency to be younger at presentation: 3.79 years, as against 5.38 years for the whole group. This was not significant by $t$ test however $(P=0.1225)$. The age at presentation was even lower if only those with $48 \mathrm{~h}$ or more delay were considered (3.29 years).

6. There was no difference in breast feeding rates or duration compared to published data on the general Australian population [4]. Seven were not breast fed at all, seven were breast fed for less than one month, 55 were breast fed for more than one month, 35 were breast fed for an unknown length of time, and on 36 there is no data at all.

7. Birth weights were compared to birth weights recorded on the neonatal database for each gestation. Birth weights are comparable to the general population. See Fig. 1.

8. There was no detectable difference in geographic distribution of children with constipation compared to normal controls, and as a result we are unable to
Fig. 1 Birth weights of children in the constipation clinic are comparable to the general population

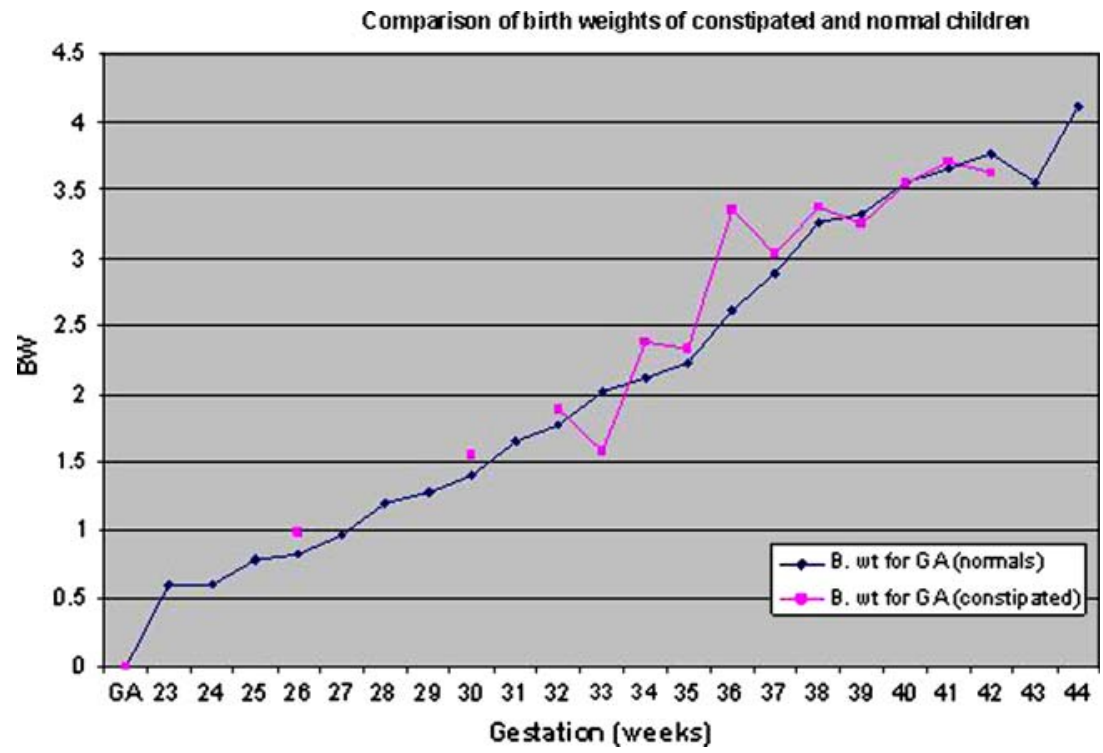


support the hypothesis that constipation is more common in the socially deprived.

\section{Discussion}

Constipation is a symptom, and not a diagnosis. Nonetheless, it is a common symptom, and one that clearly has a number of causes. There is evidence of associations of chronic constipation with a number of systemic disorders, primarily neurological in nature $[5,6]$. While the largest individual group in the constipation clinic is probably those with chronic simple constipation due to faecal withholding, there are a significant number, perhaps half, who do not respond to simple laxative and behavioural measures, and are symptomatic again years later [7]. If we accept the worst interpretation, that all those lost to follow up in our clinic were still symptomatic, then we still have a "cure" rate of over fifty percent. This is consistent with other literature [8] . It is not clear however that the "cure" will be maintained, and it is of concern that we still have an "uncured" population close to $50 \%$.

It would be of great benefit to be able to predict which children will respond to simple measures, and which will not. This study is an attempt to add to the understanding of those who will not get better with simple laxative and behavioural treatment.

In 2001 Keon and Croaker (unpublished data) presented information at the annual meeting of the BAPS making a link between social deprivation and rate of constipation in the English Midlands ("Social disadvantage as a factor in childhood constipation." C. Keon, R. Ledger, G. D. H. Croaker). Two years later we presented data at the Pacific Association of Paediatric Surgeons (PAPS) showing that a significant percentage of children who were referred for a neonatal rectal suction biopsy were still symptomatic a year later, even if the biopsy was said to be normal [9]. These two studies suggested that common effects were both congenital (Davies) and acquired (Keon). This study aims to contribute to that understanding of causes of constipation by looking back at a group of symptomatic children, and assessing relative contributions from each.

In 2004 Coerdt et al. showed that the enteric nervous system undergoes systematic changes with age. Generally, numbers of ganglion cells decrease with age. This process seems to start well before birth [10]. It may be that the children in our series with delayed passage of meconium who go on to present to our clinic have a delay in maturation of their ENS. The fact that they tend to have a younger age at presentation is consistent with this idea. The fact that delay in passage of meconium does not seem to predict slow transit on nuclear study is also consistent. It may be that some fraction of these children will revert to a normal bowel habit spontaneously in the first few years of life as the ENS continues its maturation, and, anecdotally, the senior author has indeed seen this pattern.

Whether a larger group of people with established ISTC will have a history of delay in passage of meconium is unknown, and cannot be answered from this study. Delay in passage of meconium was not a noted feature in any of the three children with abnormal nuclear transit scans discussed here. Their cases are summarised below.

1. The first patient with significant hold up above the recto-sigmoid had previous gut surgery for neonatal perforation. Figure 2 shows tracer in the whole left colon extending into the transverse colon at $96 \mathrm{~h}$. This child is now five years old with no bowel control. He was premature (ex 26 weeks), and presented with an ileal perforation. Later he had his ileostomy closed at laparotomy. He had a subsequent laparotomy for placement of a VP shunt. At recent re-laparotomy, fibrous bands were noted along the transverse colon, and it is felt that he may have had functional problems related to likely old NEC. At recent laparotomy the colon was very long, and, especially distally, very dilated. The apparently strictured area of transverse colon was resected, as well as some of the redundant left colon. For the time being he has a transverse colostomy which works well. We do not feel that this patient has ISTC.

2. The second patient is now nine months old, and was noted at antenatal ultrasound to have a megacystis. There is no mention of gut dilatation or foetal hyperechogenic bowel. There was no delay in passage of meconium according to the parents, and indeed no gut symptoms until weaning at about six months of age. From the time of weaning constipation has been an increasingly severe and intractable problem. $\mathrm{He}$ is shown to have diffuse colonic hold up on nuclear study (see Fig. 3). There was a poor urinary stream at all ages, and this persists. There is no evidence for posterior urethral valves. To rule out a smooth muscle myopathy, a full thickness open rectal biopsy was taken and subjected to electron microscopy. The EM report showed no evidence for a smooth muscle myopathy. Mitochondrial enzyme assays have not so far been done. We do not feel that this boy has an ISTC in view of the urinary tract involvement.

3. The third patient with significant colonic hold up is a school age boy who presents with soiling as a main complaint. This was unresponsive to treatment over a prolonged period. We are unaware of any alternative 


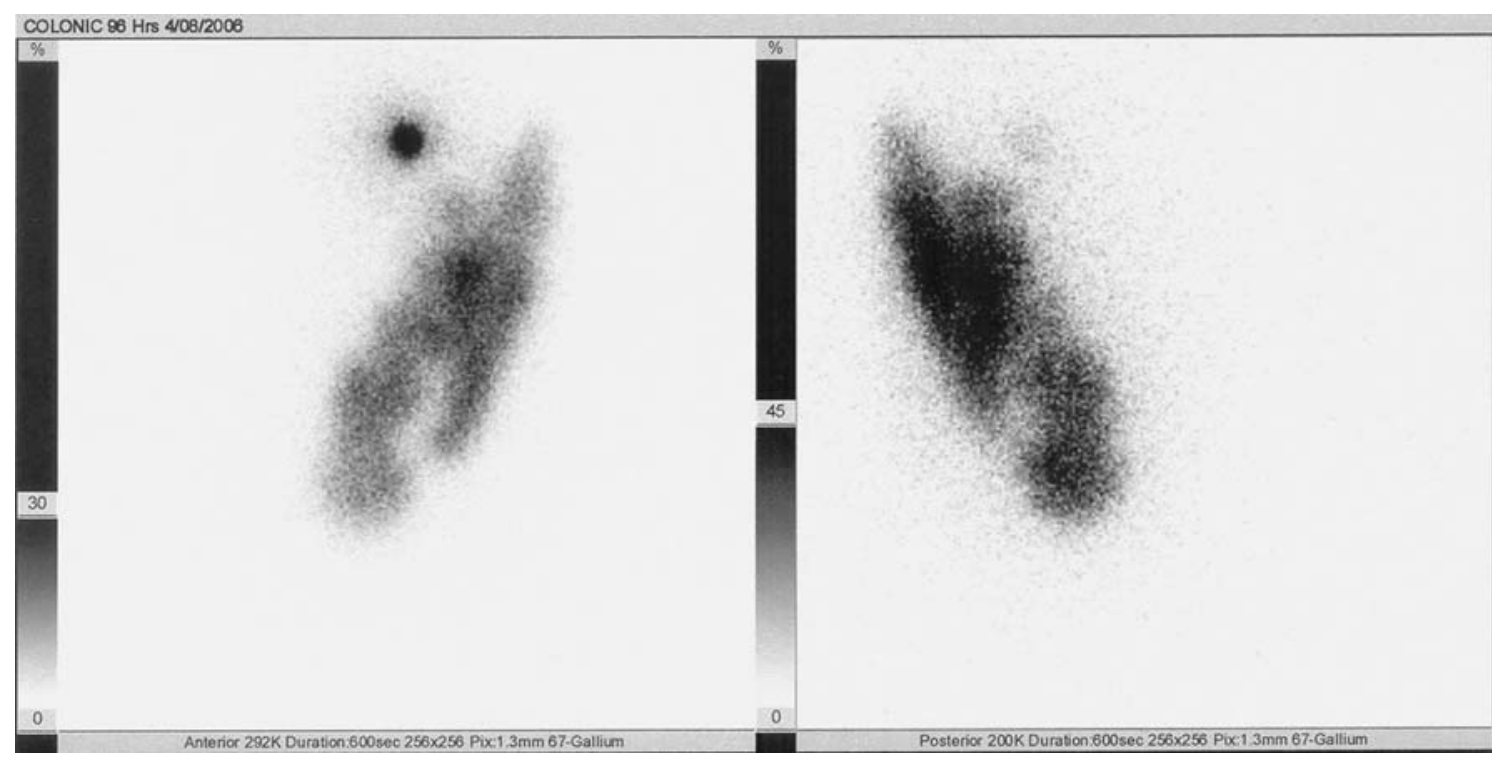

Fig. 2 Final gamma camera picture (at $96 \mathrm{~h}$ ) of the first child with significant tracer hold up, as far back as the transverse colon. This child did have thorough bowel preparation before undergoing transit study

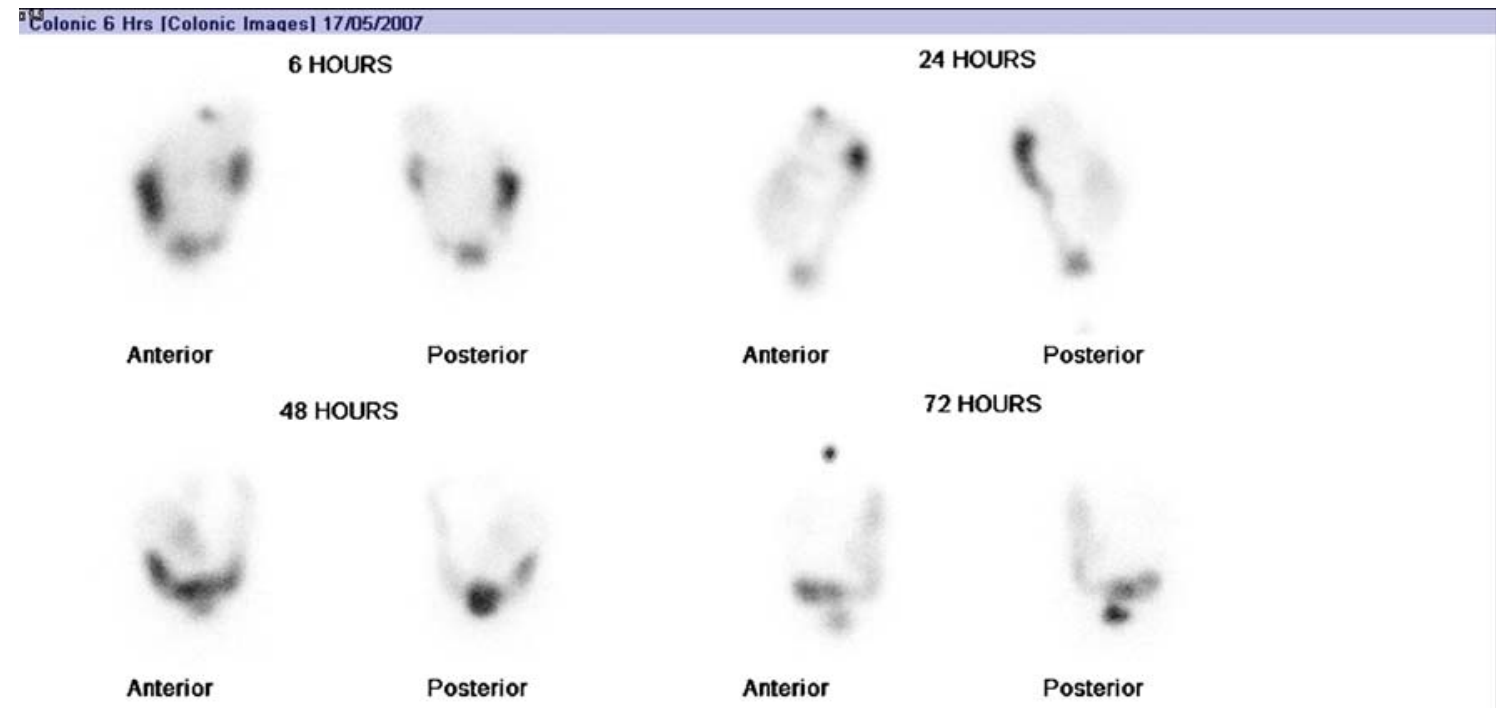

96 HOURS

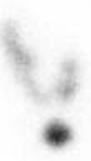

Patient 2

Anterior

Posterior

\section{Colonic Transit}

Fig. 3 The second patient has ongoing problems with bladder emptying, and diffuse slow transit of tracer around the colon. The figure shows sequential images from 6 to $96 \mathrm{~h}$

explanation for his hold up, and regard him as having true ISTC. Unfortunately his parents have not been back to discuss further treatment since his scan.
We feel therefore that the true incidence of ISTC is likely in only one of our 140 patients. It is possible that some of the patients seen during the first two and a half 
years dropped out of follow up in spite of failure of treatment, and have undiagnosed ISTC. Nonetheless, we do not think that the numbers involved are great. This is of interest given the relatively high frequency of ISTC (50 of 101 patients) reported by Cook et al. in reference 1 . They also report a slightly lower rate of functional faecal retention (22 of 101, compared to 7 of 14) than we do. Broadly our methods of scanning were similar, and we do not think that technical factors explain the different frequencies of ISTC and functional faecal retention found. Even though our patients were drawn from a specialist constipation clinic, it may still be that our population is a less selected one, and more accurately reflects the general population incidence of ISTC. If this is so, then our figures represent a more useful index of suspicion for the general clinician. A selective policy for nuclear scanning will continue to be employed in view of the low pick up rate. We do not think it would be justified to scan all patients in view of both radiation dosage, and the expense involved, given the high frequency of symptom, and the low frequency of a positive finding.

The 50\% of scanned children who have recto-sigmoid hold up, are taken to represent the group of children with functional faecal retention. This does not generally need a nuclear scan to diagnose, and indeed the scan has added little to the management of these children, except in the sense of giving the families a concrete explanation for the observed symptoms. In the relatively rare situations where it has been used, ano-rectal manometry has served this explanatory function in our clinic in the past.

The fact that delayed passage of meconium did not accurately predict either progression to nuclear transit study, or an abnormal study when one was done, is interesting. Certainly none of the three with a clearly abnormal transit study had a history of delay in passage of meconium. We now think it likely that at least some children with delayed passage of meconium who present with constipation in the first two to five years of life have a self limiting delay in maturation of the ENS which seems to get better with age. We have previously noted both the decreasing delay in passage of meconium with increasing gestation (Majeed, BAPS presentation 2004), and the findings of Coerdt [10] which support the general concept of a progressive change in structure and function of the enteric nervous system with age. Perhaps some of the problems we see are simply the result of a delay in this process?

Absence of a discernable class bias in the Canberra sample probably represents the unusual demographics of Canberra, rather than a true lack of effect. We previously noted a very striking correlation between constipation and social deprivation in a similar group of British constipation clinic children (Keon, BAPS 2001). Other authors have noted an increase in symptoms of all types in adults from more deprived backgrounds [11]. Various authors have noted a correlation between social class and breastfeeding: the more deprived having lower breast feeding rates. Our finding that breast feeding rates in our group are comparable with the national average as elsewhere reported support our negative finding on social class. We conclude that, as Canberra lacks significant social stratification compared to most parts of the world, more subtle means would be required to pick up an effect of social background, if one exists in Canberra at present.

The finding of normal rates of breast feeding in this group also tend to diminish the importance of formula feeding in contributing to later constipation. We had expected to see lower rates of breast feeding in the constipated group in view of the fact that formula is known to be associated with firmer stools than breast milk. It may be that such an effect is short lived.

Careful review of the babies' neonatal history, if necessary using the "blue book" and delivery ward records may add to our understanding of the individual patient. Although retrieval of all neonatal records was beyond the scope of this study, the information that was retrieved suggests that possibly as many as 30\% of children in the constipation clinic have a history of delay in passage of meconium greater than $24 \mathrm{~h}$, and that further children have a history of parental concern about bowel habit continuously from the neonatal period on, even if meconium was apparently not delayed.

\section{In summary}

Twenty six of 140 children had either sufficient delay in responding to treatment, or other features in the history to prompt consideration of transit study. Half these children went on to have the study, and although three had significant delays above the recto-sigmoid, only one of these has no alternative explanation for the abnormalities on study.

We conclude that ISTC as defined by nuclear transit studies, and written about by the group at Royal Children's Hospital at Melbourne [1], amongst others, has a low incidence in the community, (about $1 \%$ of children presenting from the GP or general paediatrician with constipation. Ninety five percent CI $0.02 \%-5 \%$ ) and that it cannot be easily predicted from the neonatal history. There is a substantial group of children however, who on history seem to have a congenital problem, and who are not yet well characterised. Some of these children probably have delayed maturation of the enteric nervous system, and will get better, but we expect that some of these children will form part of that group that goes on to long term constipation resistant to medication and behavioural therapy 
alike. We cannot however say that all children with a degree of delay at birth will have an unremitting problem. As the children with most delay in meconium were on average younger at presentation than the average for the clinic, it may be that some of these children represent merely the effects of a delay in maturation of the ENS and gut function, and will in fact get better. We propose to follow these children up yearly to work out the predictive power of the early childhood history.

Acknowledgments The authors wish to thank Prof. John Hutson for stimulating them to perform the transit studies. We also extend our thanks to Dr. Bruce Shadbolt for help with statistics.

\section{References}

1. Cook BJ, Lim E, Cook D, Hughes J, Chow CW, Stanton MP, Bidarkar SS, Southwell BR, Hutson JM (2005) Radionuclear transit to assess sites of delay in large bowel transit in children with chronic idiopathic constipation. J Ped Surg 40:478-483

2. Stanton MP, Hutson JM, Simpson D, Oliver MR, Southwell BR, Dinning P, Cook I, Catto-Smith AG (2005) Colonic manometry via appendicostomy shows reduced frequency, amplitude, and length of propagating sequences in children with slow-transit constipation. J Ped Surg 40:1138-1145

3. Clopper CJ, Pearson ES (1934) The use of confidence or fiducial limits illustrated in the case of the binomial. Biometrika 26:404 413
4. Donath S, Amir LH (2000) Rates of breast feeding in Australia by state and socioeconomic status: evidence from the 1995 national health survey. Breast Feed Rev 8:23-27

5. Knowles CH, Scott SM, Wellmer A, Misra VP, Pilot MA, Williams NS, Anand P (1999) Sensory and autonomic neuropathy in patients with idiopathic slow-transit constipation. Br J Surg 86:54-60

6. Abbott RD, Petrovitch H, White LR, Masaki KH, Tanner CM, Curb JD, Grandinetti A, Blanchette PL, Popper JS, Ross GW (2001) Frequency of bowel movements and the future risk of Parkinson's disease. Neurology 57(3):456-462

7. Procter E, Loader P (2003) A 6-year follow-up study of chronic constipation and soiling in a specialist paediatric service. Child Care Health Dev 29(2):103-109

8. Burnett CA, Juszczak E, Sullivan PB (2004) Nurse management of intractable functional constipation: a randomised controlled trial. Arch Dis Child 89:717-722

9. Croaker GDH, Davies B, Sideras C, Lowe J (2003) A normal neonatal rectal biopsy does not imply a normal prognosis for bowel function. Poster presentation at the 36th Annual Meeting of PAPS. Sydney

10. Coerdt W, Michel JS, Rippin G, Kletzki S, Gerein V, Muntefering H, Arnemann J (2004) Quantitative morphometric analysis of the submucous plexus in age-related control groups. Virchows Arch 444(3):239-46

11. Bytzer P, Howell S, Leemon M, Young LJ, Jones MP, Talley NJ (2001) Low socioeconomic class is a risk factor for upper and lower gastrointestinal symptoms: a population based study in 15 000 Australian adults. Gut 49:66-72 